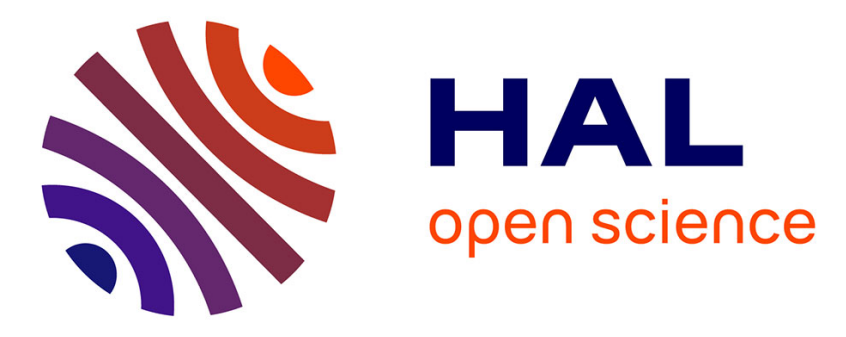

\title{
Extreme statistics in Raman fiber amplifiers : from experiments to analytical description
}

Kamal Hammani, Christophe Finot, Julien Fatome, Antonio Picozzi, Guy Millot

\section{- To cite this version:}

Kamal Hammani, Christophe Finot, Julien Fatome, Antonio Picozzi, Guy Millot. Extreme statistics in Raman fiber amplifiers: from experiments to analytical description. 12th International Conference on Transparent Optical Networks, Jun 2010, Munich, Germany. pp.We.P.13. hal-00481293

\section{HAL Id: hal-00481293 https://hal.science/hal-00481293}

Submitted on 19 Aug 2010

HAL is a multi-disciplinary open access archive for the deposit and dissemination of scientific research documents, whether they are published or not. The documents may come from teaching and research institutions in France or abroad, or from public or private research centers.
L'archive ouverte pluridisciplinaire HAL, est destinée au dépôt et à la diffusion de documents scientifiques de niveau recherche, publiés ou non, émanant des établissements d'enseignement et de recherche français ou étrangers, des laboratoires publics ou privés. 


\title{
Extreme statistics in Raman fiber amplifiers: from experiments to analytical description
}

\author{
Kamal Hammani, Christophe Finot, Julien Fatome, Antonio Picozzi and Guy Millot \\ Laboratoire Interdisciplinaire Carnot de Bourgogne, UMR 5209, 9 Av. A. Savary, Dijon, France \\ Kamal.Hammani@u-bourgogne.fr
}

\begin{abstract}
We present experimental, numerical and analytical results showing efficient emergence of rogue wave-like extreme intensity spikes during the Raman fiber amplification of a continuous wave. We show that the nonlinear pump depletion and dispersion effects strongly influence the statistical properties of the amplified signal.
\end{abstract}

Keywords: extreme events, Raman amplification, statistics.

\section{INTRODUCTION}

Optical fiber systems are well-known to provide convenient platforms in which one may investigate a large variety of fascinating fundamental nonlinear effects [1]. One of these phenomena is the emergence of extreme events that has been recently demonstrated in the supercontinuum generation initiated by high-power picosecond pulses propagating in a highly nonlinear microstructured optical fiber [2]. Other recent studies have also outlined that such statistics were not restricted to soliton-supporting systems and can be also observed in other widely spread configurations such as Raman optical amplifiers [2-4] or parametric amplifiers [5, 6] : it is shown that under the influence of a quasi-instantaneous gain and a low group velocity mismatch, fluctuations of a partially incoherent pump can be exponentially transferred to the amplified signal beam.

In the present paper, we describe experiments that reveal the significant impact of two parameters on the extreme statistics of a Raman fiber amplifier. On the one hand, we show that the initial average power of the signal and the corresponding nonlinear depletion of the pump influence the level of the extreme structures that can be detected. On the other hand, we stress the significant impact of the group-velocity difference $\delta$ between the pump and the signal. The experimental trends are confirmed by numerical simulations of the system and we also provide an analytical description of the heavily tailed statistics that appear in the probability density function of the amplified signal wave.

\section{EXPERIMENTAL RESULTS}

The system under investigation is sketched in Fig. 1a. The experimental set-up is based on commercially available telecommunication devices. The initial partially incoherent wave is delivered by a Raman laser with an output average power of $350 \mathrm{~mW}$, leading to an integrated average gain of 2. The initial continuous signal delivered by an external cavity laser is a polarized wave and its power can be adjusted between 0 and $10 \mathrm{~mW}$. Several highly nonlinear fibers (HNLF) with normal and anomalous dispersions have been tested, which allowed us to study several values of the integrated walk-off parameter, namely $\delta L$.
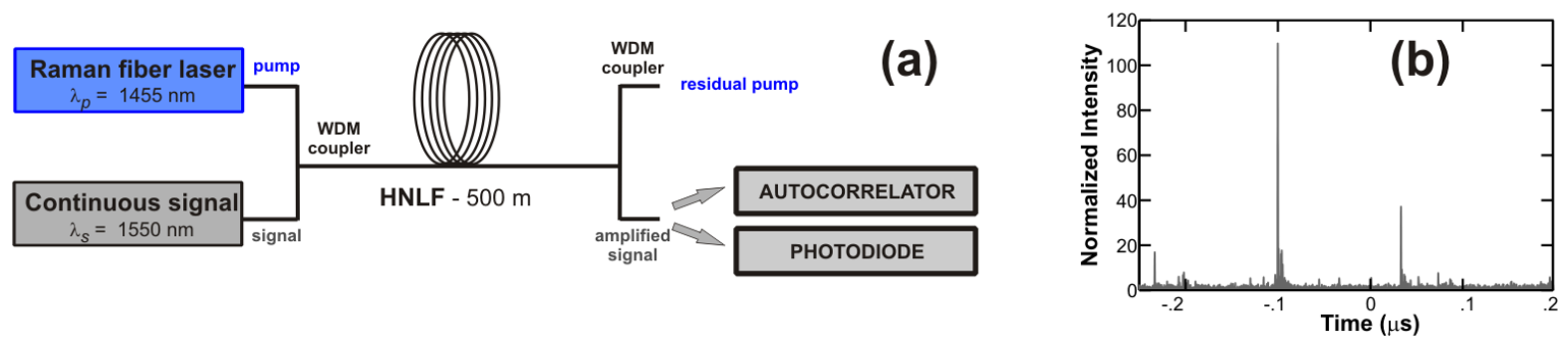

Figure 1. (a) Experimental set-up (b) Output amplified signal.

A typical measurement of an oscilloscope trace spanning 500ns is shown in Fig. $1 \mathrm{~b}$ and illustrates that the amplified signal exhibits some major intensity spikes in the temporal domain. In order to better evaluate the temporal properties of these features, an indirect but convenient way is to measure the intensity autocorrelation of the output signal [7]. From Fig. 2a, we can first notice that the width of the temporal structures is close to the typical fluctuation duration of the partially incoherent pump. However, a strong change in the contrast of the autocorrelation can be observed, suggesting significant differences in the pulse statistics. 

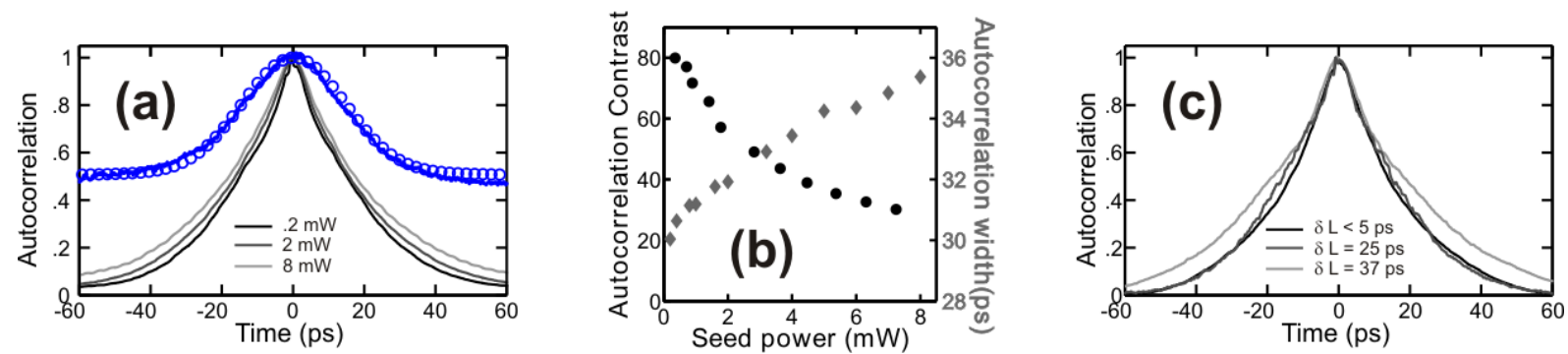

Figure 2. (a) Autocorrelation measurements obtained for various initial signal levels. The autocorrelation of the pump is plotted with a blue line and is compared with the assumption of a partially incoherent pump (circles) (b) Evolution of the autocorrelation contrast (left, black circles) and temporal width (right, grey diamonds) according to the initial signal level $\quad$ (c) Autocorrelation measurements for various values of walk-off.

Additional measurements have highlighted the strong impact of the initial signal level : an increase of the initial level leads to a continuous lowering of the contrast ratio of the autocorrelation signal (as defined as the ratio of the maximum over the minimum of the autocorrelation) combined to a temporal broadening. More quantitative results are shown in Fig. 2b. Regarding the influence of the walk-off, quite interestingly, we can note that noticeable changes in the autocorrelation only appear for values of the integrated walk-off higher than 25 ps.

\section{ANALYTICAL DESCRIPTION AND NUMERICAL RESULTS}

\subsection{Modeling of the amplification process}

Neglecting the fiber linear losses, the second order dispersion of the fiber as well as the consequences of the selfphase modulation, the evolutions of the temporal intensity profiles of the signal and pump $\left(P_{S}\right.$ and $P_{P}$ respectively) are known to be governed by the following coupled equations [8] :

$$
\left\{\begin{array}{l}
\frac{\partial P_{P}}{\partial z}=-g_{R} P_{P} P_{S} \\
\frac{\partial P_{S}}{\partial z}=g_{R} P_{P} P_{S}+\delta \frac{\partial P_{S}}{\partial T}
\end{array}\right.
$$

with $\delta=1 / v_{g P}-1 / v_{g S}$ is the walk-off parameter defined as the difference between the group velocities $v_{g S}$ and $v_{g P}$ of the signal and the pump respectively and $g_{R}$ the Raman gain experienced by the signal $\left(\sim 6.5 \times 10^{-3} \mathrm{~m}^{-1}\right)$.

A crucial point is that the pump used in our study is a partially incoherent wave $P_{P 0}$ that we model in the spectral domain by a Gaussian function with random spectral phases uniformly distributed between $-\pi$ and $\pi$. In the temporal domain this corresponds to a partially coherent wave that exhibits a stationary Gaussian statistics, whose rapid temporal fluctuations are typically of 25 ps (FWHM) [2, 3]. The corresponding probability distribution function (pdf) of the instantaneous pump power reads (see Fig. 3a) [7] :

$$
\operatorname{pdf}\left(P_{P 0}\right)=\frac{1}{\left\langle P_{P 0}\right\rangle} \exp \left(-\frac{P_{P 0}}{\left\langle P_{P 0}\right\rangle}\right)
$$

with $\left\langle P_{P O}\right\rangle$ being the average pump power, which refers in the experiment to $\left\langle P_{P 0}\right\rangle=175 \mathrm{~mW}$ (compared to the experimental value, the unpolarized nature of the pump is taken into account through a corrective factor 2 ).

\subsection{Influence of the signal average power}

In this section, we first focus on the impact of the initial continuous signal level $P_{S 0}$, neglecting the influence of the walk-off. We therefore assume $\delta=0$, which leads to the following solution of Eq. 1 :

$$
P_{S}(z, T)=\frac{P_{P 0}(T) P_{S 0}}{P_{S 0}+P_{P 0}(T) e^{-z g_{R} P_{P 0}(T)}}
$$

From the pdf of the pump (Eq. 2), we can derive the pdf of the signal after amplification [6]:

$$
p d f\left(P_{S}\right)=p d f\left(f^{-1}\left(P_{S}\right)\right) \cdot\left|\frac{d P_{P}}{d P_{S}}\right|
$$

with $f^{l}$ being the inverse function that has been calculated numerically. Results are displayed on Fig. 3a. If the depletion of the pump can be neglected, i.e. low initial signal level, an analytical form of the pdf has been obtained: 


$$
p d f\left(P_{S}\right)=\frac{1}{g z P_{S}\left\langle P_{P 0}\right\rangle}\left(\frac{P_{S 0}}{P_{S}}\right)^{\frac{1}{g_{R} z\left\langle P_{P 0}\right\rangle}}
$$

For a low amplification of the signal, the pdf obtained with and without depletion are similar and exhibit a long tail, as illustrated in Fig. 3a. However, for high signal powers, the depletion can no longer be neglected and the value of the initial pump power becomes a fundamental upper limit. This merely explains why the signal tails are asymptotically limited by the pdf of the pump. In other words, saturation of the gain strongly reduces the probability of the most extreme amplification factors.

Consequences on the autocorrelation measurement are shown on Figs. 3b and 3c. Results are in agreement with the previously described experimental trends : a major change in the contrast can be remarked: higher initial signal levels leading to lower contrasts. We can also notice that the temporal width of the autocorrelation signal broadens as the initial average powers are increased. The quantitative differences compared to the results of Fig. 2 are mainly ascribed to polarization problems and to the use in the experiments of a non-zero walk-off fiber.
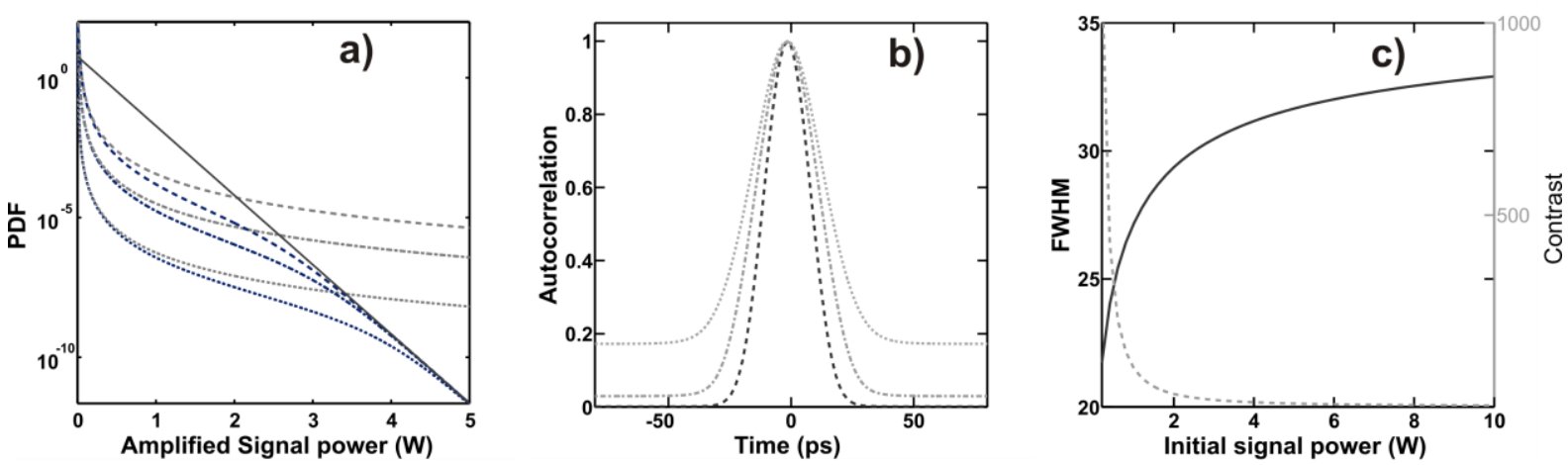

Figure 3. (a) Pdf of the amplified signal for initial average signal power of $0.2 \mathrm{~mW}, 2 \mathrm{~mW}$ and $8 \mathrm{~mW}$ (dotted, mixed and dashed lines) with (blue) and without (grey) depletion. The results are compared with the pdf of the pump (solid black). (b) Evolution of the autocorrelation signal for various signal levels. (c) Evolution of the autocorrelation temporal width (left, solid black line) and of the autocorrelation contrast (right, dashed grey line) according to the initial signal power

\subsection{Influence of dispersive properties of the fiber}

We now investigate the impact of the dispersive properties of the fiber and more specifically the impact of the walk-off between the signal and the pump. Pump depletion is neglected here, so that only low intensity initial signals are considered. From the expression of the pdf of the pump and from the following expression of the temporal intensity profile in the presence of walk-off (and without depletion) :

$$
P_{S}(z, T)=P_{S 0} \exp \left(g_{R} \int_{0}^{z} P_{P 0}\left(0, T-\delta z^{\prime}\right) d z^{\prime}\right)
$$

we can analytically derive an analytical expression of the pdf of the amplified signal:

$$
p d f\left(P_{S}\right)=\frac{1}{\left(\frac{z}{\varepsilon}-1\right) !\left(g_{R} \varepsilon\left\langle P_{P 0}\right\rangle\right)^{\frac{z}{\varepsilon}}}\left(\frac{P_{S 0}}{P_{S}}\right)^{\frac{1}{g_{R} \varepsilon\left\langle P_{P 0}\right\rangle}} \frac{1}{P_{S}} \ln ^{\left(\frac{z}{\varepsilon}-1\right)}\left(\frac{P_{S}}{P_{S 0}}\right)
$$

where $\varepsilon$ represents the correlation length of the pump as seen by the signal field due its walk-off with respect to the pump : $\varepsilon \approx \tau_{\mathrm{c}} / \delta, \tau_{\mathrm{c}}$ being the coherence time of the pump fluctuations. Fig. 4a outlines how higher walk-off reduce the tails of the signal pdf. If the differences between the pdf obtained for 0 and $50 \mathrm{ps}$ integrated walk-off are moderate, the results obtained for 100 ps value exhibit a more pronounced deviation, with a significant drop of the probability of the most intense structures.

The resulting intensity autocorrelation signals are plotted on Fig. 4(b). The temporal width of the autocorrelation and the contrast (see Fig. 4(c)) are strongly influenced by the level of walk-off. More precisely, we can observe that for values between 0 and $30 \mathrm{ps}$ (which more or less corresponds to the typical temporal extension of the pump fluctuations), those data are not too strongly impacted. Above $30 \mathrm{ps,} \mathrm{a} \mathrm{rapid} \mathrm{drop} \mathrm{of} \mathrm{the}$ contrast combined with an increase of the temporal width can be recorded, in qualitative agreement with our experimental data. 

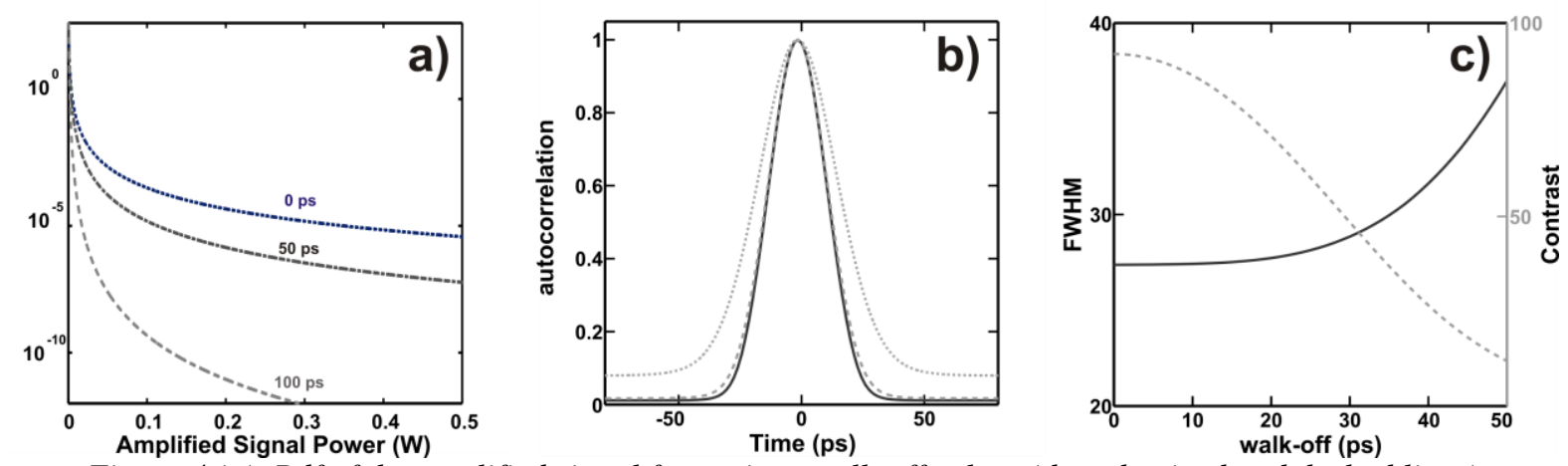

Figure 4.(a) Pdf of the amplified signal for various walk-off values (dotted, mixed and dashed lines)

(b) Intensity autocorrelation signal for walk off values of Ops, 25 ps and 50 ps (solid black, dashed, dotted lines respectively) (c) Evolution of the autocorrelation contrast (dashed grey line, right) and temporal (solid black line, left) width according to the integrated walk-off.

\section{CONCLUSIONS}

A theoretical, numerical and experimental analysis of the influence of the pump depletion and walk-off effects has allowed us to provide some clues for a better physical understanding of the amplification process taking place in discrete highly nonlinear Raman fiber amplifiers pumped in a copropagating scheme by a partially incoherent wave. Depletion and walk-off can both substantially reshape the statistical properties of the output signal, the generation of the most intense optical peaks being stimulated by negligible depletion effect combined with low walk-off values. On the contrary, reducing output fluctuations requires a high walk-off and/or saturation of the amplifier. Such conclusions derived by a temporal domain analysis of the probability distribution functions of the instantaneous power are in full agreement with previous results based on the evaluation of the relative intensity noise evolution during the amplification process [9].

In the present proceeding, we have mainly focused the discussion on the temporal aspects. However, the optical spectrum is also highly affected, experiencing a significant expansion caused by cross phase modulation of the pump on the signal. Additional experimental and numerical results describing in more details this effect will be presented.

\section{ACKNOWLEDGEMENTS}

We would like to thank J.M Dudley for illuminating discussions. This work was supported by the Agence Nationale de la Recherche (ANR MANUREVA and PERSYST II projects: ANR-08-SYSC-019 and ANR-07TCOM-014) and by the Conseil Régional de Bourgogne.

\section{REFERENCES}

[1] G. P. Agrawal, Nonlinear Fiber Optics, Third Edition. San Francisco, CA: Academic Press, 2001.

[2] K. Hammani, C. Finot, J. M. Dudley, and G. Millot, "Optical rogue-wave fluctuations in fiber Raman amplifiers," Opt. Express, vol. 16, pp. 16467-16474, 2008.

[3] C. Finot, K. Hammani, J. Fatome, J. M. Dudley, and G. Millot, "Selection of extreme events generated in Raman fiber amplifiers through spectral offset filtering," IEEE J. Quantum Electron., vol. 46, pp. 205-213, 2009.

[4] D. Borlaug, S. Fathpour, and B. Jalali, "Extreme value statistics in silicon photonics," IEEE Photon. J., vol. 1, pp. 33-39, 2008.

[5] K. Hammani, C. Finot, and G. Millot, "Emergence of extreme events in fiber-based parametric processes driven by a partially incoherent wave," Opt. Lett., vol. 34, pp. 1138-1140, 2009.

[6] S. G. Murdoch and Y. Xu, "Gain statistics of a fiber optical parametric amplifier with a temporally incoherent pump," Opt. Lett., 2010.

[7] J. W. Goodman, Statistical optics: John Wileay and Sons, 1985.

[8] C. Headley and G. P. Agrawal, Raman amplification in fiber optical communications: Academic Press, 2005.

[9] C. Fludger, V. Handerek, and R. J. Mears, "Pump to signal RIN transfer in Raman fiber amplifiers," $J$. Lightw. Technol., vol. 19, pp. 1140-1148, 2001. 